\title{
Diversa
}

\section{Im Mai 1996 feierte Dr. Günter SCHMIDT seinen 70. Geburtstag}

Schriftleitung und Redaktion gratulieren nachträglich und wünschen dem Jubilar auch weiterhin Gesundheit und viel Freude im persönlichen und arachnologischen Bereich.

\section{Suche: Trichoncus saxicola- Fundpunkte}

Aufgrund der weitgehend unbekannten Verbreitung von Trichoncus saxicola, suche ich Fundmeldungen. Wer mir helfen möchte, wendet sich bitte an folgende Adresse:

Martin KREUELS, Theodor-Heuss-Str. 32, D-48167 Münster, Tel.: 0251/617361,FAX: 0251/838352,E-mail:Kreuels@Uni-Muenster.de

\section{Suchaufruf: Spinnen des Moselgebietes}

Für meine Diplomarbeit suche ich alle Daten zur Spinnenfauna des Moselgebietes und Arbeiten, die sich mit dem Thema Spinnen an Ufern beschäftigen. Anfallende Kosten werden erstattet. Johannes GÜNNEBERG, Fröbelstr. 48, D-50823 Köln Tel.: 0221/5101678

\section{Call for material}

Any possible material of SE-Asian Clubiona is requested for my further clubionid study, especially from Indonesia, Thailand, Burma, etc. I am ready to sort out all necessary specimens from mixed collections. Both identified and indetermined material is of my interest. Details are to be discussed. Kirill G.MIKHAILOV, Zoological Museum of Moscow State University. Bolshaya Nikitskaya Street 6, Moscow 103009 Russia. FAX: (+7-095) 203-2717, E-mail: arthro@glas.apc.org 